\title{
ROR1 Positive
}

National Cancer Institute

\section{Source}

National Cancer Institute. ROR1 Positive. NCI Thesaurus. Code C142123.

An indication that ROR1 expression has been detected in a sample. 